Copyright (C) 2014 IEEE. Personal use of this material is permitted. Permission from IEEE must be obtained for all other uses, in any current or future media, including reprinting/republishing this material for advertising or promotional purposes, creating new collective works, for resale or redistribution to servers or lists, or reuse of any copyrighted component of this work in other works. 


\title{
Evaluation Study and Results of Intelligent Pedagogical Agent-led Learning Scenarios in a Virtual World
}

\author{
M. Soliman*, and C. Guetl ${ }^{*}, * *$ \\ * Graz University of Technology/IICM, Graz, Austria \\ ${ }^{* *}$ Curtin University/School of Information Systems, Perth, Austria \\ muhamed.soliman@gmail.com, christian.guetl@iicm.tugraz.at
}

\begin{abstract}
The use of intelligent pedagogical agents (IPAs) has shown to provide positive learning results and new learning possibilities. The IPA has specific importance in immersive learning environments to enable $24 / 7$ availability, offer a learning companion opportunity, and increase learner engagement. In that regard, a proof of concept prototype implementation of an intelligent pedagogical agent (IPA) in the Open Wonderland virtual world environment was created. This paper reports a qualitative evaluation study and experiment performed by a team of six experts in relevant areas of expertise. Those areas include cognitive science, computer science, e-education, and virtual worlds. The experiment studied key prototype components in relation to four learning scenarios with distributed control between the learner avatar and the pedagogical agent to answer questions relevant to their effect on learning attributes of such as motivation, engagement, and the learning experience. Given the qualitative nature of the experiment, the paper also analyzes and reports results relevant to expert input of how the prototype can better contribute to future pedagogical agent realizations and the impact on learning enhancement.
\end{abstract}

\section{INTRODUCTION AND BACKGROUND}

In attempts to discover various methods for new technology enhanced learning, immersive environments provide interesting properties for learning support. Those properties include 3D visualization, collaborative learning, and more to add for convenience, remote access, and cost effectiveness, [11]. A particular implementation of immersive environments is virtual worlds with examples such as Open Wonderland [1]. Virtual World environments are accessible through internet browsers while representing each user as an avatar. The user avatar can thus roam in the $3 \mathrm{D}$ space and perform available learning activities alone or in collaboration with others who are online.

An important challenge in virtual worlds is learning support. Given the convenience of the time and location, the immersive environment can be occupied with many users. It can be infeasible to achieve 24/7 human learning support through avatars. Furthermore, learner who cannot find peers to collaborate or login to an inhabited world can find it uninteresting to proceed.
The use of intelligent pedagogical agents (IPAs) has shown to provide positive learning results and new learning possibilities. IPAs are lifelike characters that can be thought of as points of interaction between the learner and an immersive environment to provide learning support, [2]. There has been research in the area of pedagogical agents for long time from different sions [2][4][5][6][12]. The motivation for incorporating IPAs in an immersive environment provide interesting properties of 24/7 learning support, provide learning peers, and offer intelligent learning support.

The goals of the IPA are pedagogical oriented to increase learner engagement and interactivity in the virtual world and provide effective learning services while monitoring learner progress. To discover feasible interaction possibilities between the learner and the IPA in learning scenarios, in the learning context, a simulation was achieved to show interaction pattern [5]. Furthermore, adding intelligence abilities the IPA was also studied in [6].

Realizing IPA has been reported in [4] providing a prototype of an IPA in the Open Wonderland virtual world to offer different types of services. In order to achieve it, a Non-Player Character (NPC), a multi-modal communication module, and supporting elements were developed and amended with Bot Chat module. The purpose of the multimodal communication module is to enable interaction with the IPA. Furthermore, a voice chat was added through voice synthesis tool, [9]. The developed prototype in reference to the performed simulation provided further details of interaction in the implementation of Open Wonderland virtual world. This formed different learning scenarios, in each of which the pedagogical agent provides specific learning services with objectives.

In order to assess the prototype and to evaluate its impact on the learning experience in the virtual world, an evaluation experiment is required. There were two approaches to obtain the evaluation: through user evaluation or through expert evaluation. The second approach, that is selected, is in relation to the method of performing a qualitative experiment to obtain experts opinion on various aspects. 


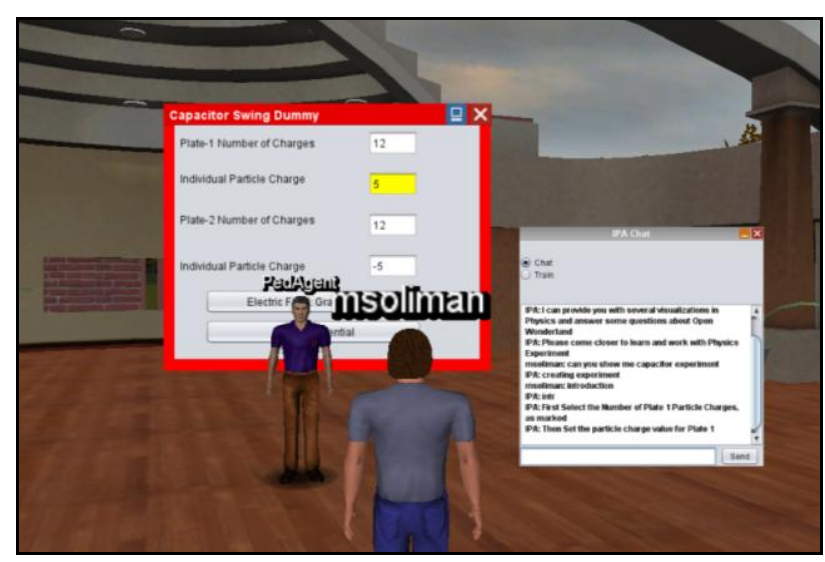

Figure 1. IPA interacts with a learner avatar on an experiment object. A text chat tool is used for communication.

\section{EXPERIMENT SETUP}

\section{A. Research Questions and Design}

The following hypotheses are formed to be checked in the experiment:

H1: The IPA, in the provided setting, provides appropriate mechanisms for increasing learner engagement in the virtual world.

H2: The IPA, in the provided setting and through awareness of the learning object, furnishes important guided instruction as an approach for enhancing the learning experience.

H3: The IPA contributes positively to learner motivation through guidance and task-completion support.

H4: The IPA provides the learner with an appropriate feedback mechanism that is positive to the learning experience.

H5: The pedagogical agent positively contributes to the learning experience in the virtual world setting.

In addition, the study has the following objectives:

O1: Evaluating and validating hypotheses, H1:H5.

O2: Evaluating appropriateness and alternatives of the design elements such the text chat tool.

O3: Evaluating how can the prototype implementation potentially support the IPA concept.

\section{B. Apparatus}

To measure hypotheses and objectives, the prototype setting is used forming four learning scenarios in Open Wonderland. Participants refer to experiencing the following scenarios upon filling a questionnaire.

- IPA proximity. Captures learner intention to start learning activity as well as IPA attempt to engage the learner through two proximity zones.

- Using multi-modal communication. Learner to IPA interaction in either text or voice. Answers to questions are given through an AIML ule, [4][10].
- Tutoring. The IPA tutors a sample simulation experiment.

- Activity feedback. The IPA observes and provides feedback to the learner in relation to the learning object visual module.

\section{Participants}

Given the qualitative nature of the experiment, the study incorporated six experts in various fields of relevant specialties of computer science, cognitive science, eeducation, and virtual worlds. Table I shows areas of specialties and distribution. Some of the experts are in research of more than one field. Two of the participants are in the Masters program while the other four are in the Ph.D. program and with Master's degree. The age of the participants are in the range of 24-44 with (Mean $=30.3 \&$ $\mathrm{SD}=7.2)$. Three $(50 \%)$ of the participants are females and three $(50 \%)$ are males.

TABLE I. EXPERTS AREAS OF SPECIALTY AND RELEVANCE TO THE STUDY.

\begin{tabular}{|l|l|c|}
\hline \multicolumn{2}{|c|}{ Area, relevance, and number of participants (\%) in each area. } \\
\hline \multicolumn{1}{|c|}{ Relevance } & $\begin{array}{c}\text { Number } \\
(\%)\end{array}$ \\
\hline $\begin{array}{l}\text { Cognitive } \\
\text { Science }\end{array}$ & $\begin{array}{l}\text { Thought processes of the learner, expo- } \\
\text { sure to artificial methods for e-education. }\end{array}$ & $3(50 \%)$ \\
\hline E-education & $\begin{array}{l}\text { Experience with tools and methods for } \\
\text { electronic instruction. }\end{array}$ & $4(67 \%)$ \\
\hline $\begin{array}{l}\text { Computer } \\
\text { Science }\end{array}$ & Facilitating methods of IPA foundations. & $5(83 \%)$ \\
\hline $\begin{array}{l}\text { Virtual } \\
\text { Worlds }\end{array}$ & $\begin{array}{l}\text { Main immersive environment the peda- } \\
\text { gogical agent operates in. }\end{array}$ & $5(83 \%)$ \\
\hline
\end{tabular}

\section{Procedure}

The procedure of the study includes four main steps for each participant, as follows:

1. Filling pre-questionnaire by the participant

2. Introduction to the system

3. Participant performs four IPA supported scenarios

4. Filling post-questionnaire

\section{E. Pre and Post Questionnaires}

The purpose of the pre-questionnaire is to obtain demographic data and prior expert knowledge in the relevant areas as discussed in Section C. The contents of the postquestionnaire measures are for the hypotheses and the objectives. The majority of the questionnaire questions are Likert based except questions 2,3,21. The postquestionnaire also obtains qualitative opinions through open questions. Table II shows Likert and multiple choice questions of the post-questionnaire. 
TABLE II. POST-QUESTIONNAIRE QUESTIONS.

\begin{tabular}{c|c} 
Likert Question \\
(1-Strongly agree, 2-Agree, 3-Neutral, 4-Disagree, 5-Disagree)
\end{tabular}

1 I think using the question answering tool supports the learning activity.

4 I felt that IPA gestures are helpful to the learning experience.

5 I thought that the voice capability of the pedagogical agent is important.

6 I felt that the pedagogical agent has some sort of intelligence.

7 I find the method of detecting avatar proximity is good to determine learner intention to run the activity.

8 The proximity scenario helps to increase learner attention to the learning activity in the virtual world.

9 In tutoring and activity feedback scenarios, the pedagogical agent increases learner attention to the learning task.

10 I felt that the IPA encourages me to interact more with it.

11 I felt the IPA encourages the learner user to interact more with the environment (learning object).

12 I think the tutoring scenario by the IPA contributes positively to the learning experience.

13 I think the observing and providing activity feedback scenario is important to the learning experience.

14 I think that the IPA support to learning by doing improves the learning experience in this virtual world setting.

15 Overall, the IPA contributes tactics that positively support the learning activity in a virtual world

16 I felt that the IPA furnished guided instruction with the learning task, as an approach for enhancing the experience.

17 Through the task-completion support, the IPA helps improve learner motivation.

18 I learned something new about pedagogical agents.

19 I think the IPA is an essential learning component to an immersive learning environment.

20 The virtual world can become more interesting to the learner being more inhabited with pedagogical agents.

Multiple Choice Questions

2 Which tool is preferable for activating pedagogical agent functions and obtaining its responses?

(Choices: Text Chat tool, Traditional 2D menu, Other)

3 Having both the text and voice chat options are important to the user. (Choices: Yes, No)

21 I view that the virtual world learner user will prefer to learn with a similar pedagogical agent....(Choices: None of the time, Some of the time, Most of the time, All of the time)

\section{RESULTS}

\section{A. Pre-Questionnaire Results}

Participants answered the open question "Which application types you find a virtual world useful for" with different application types of collaboration, simulation and visualization, language learning, scientific research, and games. Their views on the advantages of virtual worlds for e-education included: flexible location, visualization in $3 D$, spatial information, immersion, freedom, collaboration, simulation of history, physics, learning together for physically apart students, and meeting native speakers for language learners. In response to stating disadvantages of using a virtual world for e-education, the following are included: The demanding requirements of good internet connection and graphics, usability issues, being hard for new users, user interface challenges, not always up to date, can seem to be complicated for users with low IT skills.

$33 \%$ only of the participants indicated prior knowledge about pedagogical agents (Agree), 50\% are neutral, and one participant $(16.7 \%)$ doesn't have pedagogical agent knowledge (disagree). One participant $(16.7 \%)$ agree to have prior experience with pedagogical agents in virtual worlds, one is neutral (16.7\%), 50\% disagree, and (16.7\%) strongly disagree. They indicated expectations on the IPA as: to provide guidance and hints, explanations and repetitions, being intuitive, easy to use, and flexible. They see advantages of IPA in the virtual world to: help in the learning efforts, help to perform a task, having more knowledge, provide easy instructions, support learning at any place and any time. Indicated views on disadvantages included: distraction of the task, and of possessing less in personality.

\section{B. Post-Questionnaire Results}

The purpose of the post-questionnaire is to measure for hypotheses and objectives. The majority of choice questions are in Likert scale [7] (1-Strongly agree, 2Agree, 3-Neutral, 4-Disagree, 5-Strongly disagree), except for three questions. The post-questionnaire also includes open questions to obtain qualitative opinion relevant to hypotheses and prototype design elements and how to improve them. Table II shows choice questions in the post-questionnaire. While there is a disagreement in specific areas of research for calculating the mean for Likert items, the reason is due to assumptions on equal distances among the scale items. Thus, further measures are added including the Standard Deviation (SD), the Mode (most repeating response), and the frequency for each response, [8]. Table III summarizes the responses to each Likert question giving the various measures used.

\section{ANALYSIS OF RESULTS AND HYPOTHESES EVALUATION}

Each hypothesis is measured based on different related questions from the questionnaire. Please refer to Table II for the specific question. In addition, open questions provide qualitative input.

A. H1: The IPA, in the provided setting, provides appropriate mechanisms for increasing learner engagement in the virtual world.

This hypothesis is measured through questions 8-11. On the overall, participants agree on questions relevant to this hypothesis. A combined score of $(\mathrm{M}=1.95, \mathrm{SD}=$ 0.75 , Mode $=2$ ) is obtained assuming equal weights. On the overall $83.5 \%$ agree, $4 \%$ disagree, and $12.5 \%$ neutral.

Open questions responses such as "You feel personally involved when a chat starts", "Very nice approach to make learners curious", "You feel personally involved when a chat starts" indicate support to the hypothesis in relation to specific elements of the prototype. 
TABLE III. LIKERT QUESTIONS: RESPONSES AND BASIC CALCULATIONS

\begin{tabular}{|c|c|c|c|c|c|c|c|c|}
\hline \multirow{2}{*}{$Q$} & \multirow{2}{*}{ Mean } & \multirow{2}{*}{$S D$} & \multirow{2}{*}{ Mode } & \multicolumn{5}{|c|}{$\begin{array}{c}\text { Percentage Distribution } \\
\text { 4-Disagree, } 5 \text {-Disagree }\end{array}$} \\
\cline { 5 - 9 } & & & & $(1)$ & $(2)$ & $(3)$ & $(4)$ & $(5)$ \\
\hline 1 & 2.17 & 1.17 & 1 & $33 \%$ & $33 \%$ & $17 \%$ & $17 \%$ & $0 \%$ \\
\hline 4 & 3.00 & 1.10 & 3 & $0 \%$ & $33 \%$ & $50 \%$ & $0 \%$ & $17 \%$ \\
\hline 5 & 2.33 & 1.21 & 1 & $33 \%$ & $17 \%$ & $33 \%$ & $17 \%$ & $0 \%$ \\
\hline 6 & 3.33 & 1.21 & 2 & $0 \%$ & $33 \%$ & $17 \%$ & $33 \%$ & $17 \%$ \\
\hline 7 & 1.33 & 0.52 & 1 & $67 \%$ & $33 \%$ & $0 \%$ & $0 \%$ & $0 \%$ \\
\hline 8 & 1.50 & 0.55 & 2 & $50 \%$ & $50 \%$ & $0 \%$ & $0 \%$ & $0 \%$ \\
\hline 9 & 2.00 & 0.63 & 2 & $17 \%$ & $67 \%$ & $17 \%$ & $0 \%$ & $0 \%$ \\
\hline 10 & 2.33 & 1.03 & 2 & $17 \%$ & $50 \%$ & $17 \%$ & $17 \%$ & $0 \%$ \\
\hline 11 & 2.00 & 0.63 & 2 & $17 \%$ & $67 \%$ & $17 \%$ & $0 \%$ & $0 \%$ \\
\hline 12 & 1.83 & 1.17 & 1 & $50 \%$ & $33 \%$ & $0 \%$ & $17 \%$ & $0 \%$ \\
\hline 13 & 1.50 & 0.84 & 1 & $67 \%$ & $17 \%$ & $17 \%$ & $0 \%$ & $0 \%$ \\
\hline 14 & 1.67 & 1.21 & 1 & $67 \%$ & $17 \%$ & $0 \%$ & $17 \%$ & $0 \%$ \\
\hline 15 & 2.17 & 0.98 & 3 & $33 \%$ & $17 \%$ & $50 \%$ & $0 \%$ & $0 \%$ \\
\hline 16 & 2.33 & 1.03 & 2 & $17 \%$ & $50 \%$ & $17 \%$ & $17 \%$ & $0 \%$ \\
\hline 17 & 2.00 & 0.63 & 2 & $17 \%$ & $67 \%$ & $17 \%$ & $0 \%$ & $0 \%$ \\
\hline 18 & 1.67 & 0.52 & 2 & $33 \%$ & $67 \%$ & $0 \%$ & $0 \%$ & $0 \%$ \\
\hline 19 & 2.33 & 0.82 & 3 & $17 \%$ & $33 \%$ & $50 \%$ & $0 \%$ & $0 \%$ \\
\hline 20 & 1.50 & 0.55 & 2 & $50 \%$ & $50 \%$ & $0 \%$ & $0 \%$ & $0 \%$ \\
\hline
\end{tabular}

B. H2: The IPA, in the provided setting and through awareness of the learning object, furnishes important guided instruction as an approach for enhancing the learning experience.

This hypothesis is checked through questions $12,13,14,16$. On the overall participants agree on questions relevant to this hypothesis. A combined score of (M $=1.83, \mathrm{SD}=1.05$, Mode $=1)$ is obtained assuming equal weights. On the overall, $50 \%$ strongly agree, $29 \%$ agree, $8.3 \%$ are neutral, and $12.5 \%$ disagree in combining with equal weights. In Agree/Disagree calculation, overall 79\% agree. Accordingly, it is approximated to support this hypothesis with Agree.

Participants indicated support to this hypothesis with responses "Hints/Visual Hints (yellow marks) where was the focus" are liked, "The IPA telling me about right and wrong inputs. Participants showed interest of the importance of providing more detailed and specific feedback by the IPA as for improvement suggestion.

\section{H3: The IPA contributes positively to learner motivation through guidance and task-completion support.}

This hypothesis is checked through questions 8,17 , and 20. On the overall, participants agree on questions of this hypothesis with combined score of $(\mathrm{M}=1.67, \mathrm{SD}=$ 0.59 , Mode $=2$ ). On the overall $100 \%$ agree, if excluding one neutral opinion. Accordingly, this hypothesis is supported with Agree.

Participants indicated strong support to this hypothesis with several responses as a good approach such as "Fits very well", providing guidance, It is not forcing the learner", "By motivating and by giving hints \& how to solve the simulation", and more.

\section{H4: The IPA provides the learner with an} appropriate feedback mechanism that is positive to the learning experience.

This hypothesis is checked through questions $9,13,16$. On the overall participants agree on questions relevant to this hypothesis. A combined score of $(\mathrm{M}=1.9, \mathrm{SD}=0.87$, Mode $=2$ ). On the overall $77.78 \%$ agree, $5.56 \%$ disagree, and $16.67 \%$ are neutral. Accordingly, it is approximated to support this hypothesis with Agree.

Participants indicated support to the hypothesis through open questions such as "Feedback helps during experiments", "Wrong answers (actions) gets corrected immediately". They also provided important area of improvement in this aspect to provide specific what went wrong, and how to perform it correctly.

\section{E. H5: The pedagogical agent positively contributes to} the learning experience in the virtual world setting.

This hypothesis is checked through questions 12-17 to give an overall result of $(\mathrm{M}=1.91, \mathrm{SD}=0.97$, Mode $=1)$ with equal weights. On the overall $41.67 \%$ strongly agree, $33.33 \%$ agree, $8.33 \%$ disagree, and $16.67 \%$ are neutral. Accordingly, it is approximated to support this hypothesis with Agree.

Responses to open questions in relation to particular elements also support this general hypothesis. The hypothesis is also related to Q21, 83\% some of the time while $17 \%$ indicated most of the time choice to conclude its positive contribution to learning experiences in the virtual world, but not in all cases.

\section{F. Measuring Prototype Tools Appropriateness to the Learning Activity}

Questions 1-5 are relevant to tools appropriateness to the learning activity; see Table II for questions and Table III for results. Experts in general agreed to the importance of the text chat tool and preference over a traditional 2D menu to interact with the pedagogical agent, and activate its functions. Less importance is then given to the IPA gestures. Furthermore, suggestions are provided to enhance the text chat tool through auto-completion features and action recommendation.

\section{G. How the Prototype Supports the IPA Concept}

Questions 18-21 are relevant to generic statements about the prototype in relation to the IPA concept in general; please see Table III for calculations for each question. In general, the prototype has provided input to have something new about pedagogical agents with agree $(\mathrm{M}=$ $1.67, \mathrm{SD}=0.52$, Mode $=2$ ), see Fig. 2. Participants either agree or strongly agree to support IPA influence on giving users the feel of the virtual world being more inhabited (M 
$=1.5, \mathrm{SD}=.55$, Mode $=2$ ), Fig. 3 shows the resulting calculation of this question of $50 \%$ strongly agree and $50 \%$ agree.

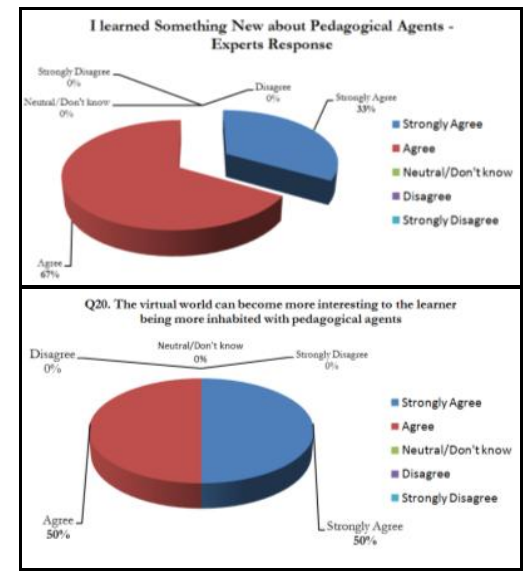

Figure 2. Results of questions Q18 and Q20 which are related to the IPA concept.

\section{H. Further Study Conclusions and Outlook}

The study can thus shed light into most important aspects of the learning scenarios and design elements of the prototype. One of the most found scenarios is the proximity scenario as the IPA attempts to reason about learner intentions for performing activity and motivates to get engaged in the possible activity. The relevant question $(\mathrm{Q} 7, \mathrm{Q} 8)$ had results of $(\mathrm{M}=1.33, \mathrm{SD}=0.52$, Mode $=1)$ and $(\mathrm{M}=1.5, \mathrm{SD}=0.55$, Mode $=2)$ and with $100 \%$ agreement, see Tables II, III.

Regarding prototype elements importance, experts indicated the importance of the text chat module as a key component for chat with the IPA. They also suggested elements of enhancement with adding auto-complete feature and action recommendations by the pedagogical agent.

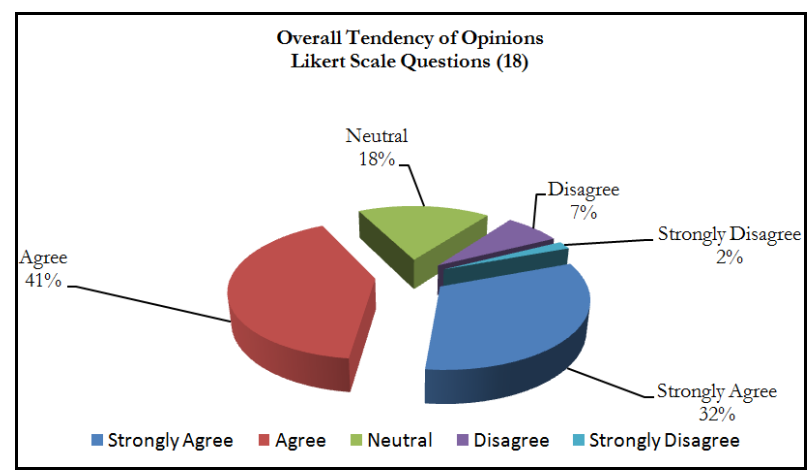

Figure 3. Overall tendency of opinions combined score.

\section{CONLUSION}

The paper described an experiment setup and results to evaluate intelligent pedagogical agent learning scenarios in a virtual world. The purpose is to evaluate relevant hypotheses to pedagogical agent influence on the learner experience in a virtual world. The study had a qualitative nature by six expert participations from cognitive science, computer science, e-education, and virtual worlds. Prequestionnaire and post-questionnaires are filled by each participant. The questions were in relation to four learning scenarios by the pedagogical agent in the Open Wonderland virtual world environment. Most questions are Likert questions and open ended questions to capture general opinions. On the overall, there was an agreement on the following hypotheses:

- The IPA provided appropriate mechanisms for increasing learner's engagement in the virtual world.

- The IPA through awareness of the learning object, furnished important guided instruction as an approach for enhancing the learning experience.

- The IPA contributed positively to learner motivation through guidance and task-completion support.

- The IPA provided the learner with an appropriate feedback mechanism that is positive to the learning experience.

On the overall, the pedagogical agent positively contributes to the learning experience in the virtual world setting was also validated. The results also show particular strong validation to learner engagement addition by the pedagogical agent and the importance of the proximity learning scenario. Through the open questions, the study suggests strong areas of the prototype including a text chat module and suggested further improvements.

\section{ACKNOWLEDGMENT}

(The authors would like to thank) Appreciation goes to (the anonymous expert evaluators) Alexander Nussbaumer, Johanna Pirker, Lisa Tomes, Michael Bedek, Patrick Weghofer, and Simone Kopeinik from TU-Graz for contributing their time and effort to provide valuable contribution to the study.

\section{REFERENCES}

[1] OpenWonderland, http://openwonderland.org/, Online, Accessed March. 15, 2014.

[2] M. Soliman and C. Guetl, "Intelligent pedagogical agents in immersive virtual learning environments: a review," Proceedings of $33^{\text {rd }}$ International Convention on Information and Communication Technology, Electronics and Microelectronics, MIPRO, May 24-28 2010, Opatija, Croatia.

[3] J. Kaplan and N. Yankelovich, "Open Wonderland: an extensible virtual world architecture," IEEE Internet Computing, 2011, 3845.

[4] M. Soliman and C. Guetl, "Implementing intelligent pedagogical agents in virtual worlds: tutoring natural science experiments in OpenWonderland," The IEEE Global Education Conference, EDUCON 2013, March 12-15, Berlin, Germany.

[5] Soliman, M. and C. Guetl, "Simulating interactive learning scenarios with intelligent pedagogical agents in a virtual world through BDI-based agents. International Journal of Engineering Pedagogy (iJEP), vol. 3, no. 2., pp. 41-47, April, 2013.

[6] M. Soliman and C. Guetl, "Evaluation of intelligent agent frameworks for human learning in virtual worlds," International Journal of Engineering Pedagogy (iJEP), vol. 1, no. 3, pp. 45-48 Oct. 2011.

[7] R. Likert, "A technique for the measurement of attitudes. Archives of Psychology," vol. 22, no. 140, pp. 1-55, 1932. 
[8] H. N. Boone, Jr. and D. A. Boone, "Analyzing Likert data," Journal of Extension, vol. 50, no. 2, 2012.

[9] Mary Text To Speech, http://mary.dfki.de/, Online, Accessed March 15, 2014.

[10] AIML: Artificial Intelligence Markup Language, http://www.alicebot.org/aiml.html, Online, Accessed March 15, 2014
[11] Immersive Education, http://immersiveeducation.org/, Online, Accessed March 15, 2014.

[12] W. Johnson, J. Rickel, R. Stiles, and A. Munro, A., "Integrating Pedagogical Agents into Virtual Environments," Teleoperators and Virtual Environments. Vol. 7(6), pp. 523-546. 1998. 